\title{
Early Oscillation Detection for DC/DC Converter Fault Diagnosis*
}

\author{
(Abstract for AIAA $9^{\text {th }}$ IECEC, September 1-3, 2011, San Diego, CA) \\ Bright L. Wang \\ MEI Technologies Inc. \\ 7404 Executive Place, Suite 500 \\ Seabrook, MD 20706 \\ bright.l.wang@nasa.gov
}

The electrical power system of a spacecraft plays a very critical role for space mission success. Such a modern power system may contain numerous hybrid DC/DC converters both inside the power system electronics (PSE) units and onboard most of the flight electronics modules. One of the faulty conditions for DC/DC converter that poses serious threats to mission safety is the random occurrence of oscillation related to inherent instability characteristics of the DC/DC converters and design deficiency of the power systems. To ensure the highest reliability of the power system, oscillations in any form shall be promptly detected during part level testing, system integration tests, flight health monitoring, and on-board fault diagnosis. The popular gain/phase margin analysis method is capable of predicting stability levels of DC/DC converters, but it is limited only to verification of designs and to part-level testing on some of the models. This method has to inject noise signals into the control loop circuitry as required, thus, interrupts the DC/DC converter's normal operation and increases risks of degrading and damaging the flight unit [1]

A novel technique to detect oscillations at early stage for flight hybrid DC/DC converters was developed. The technique utilizes a power spectral analysis method to detect early-stage oscillation signals buried in a wide range of input voltage noise frequencies of the converter and to identify the oscillation signal based on RF signal amplitude/frequency modulation (AM/FM) theories [2]. This technique provides a useful tool to diagnose real-time stability problems for spacecraft power systems consisting of various DC/DC converters. To monitor and detect the oscillation, one can simply connect a test probe of a spectral analyzer to the power input of an operating DC/DC converter. A power spectrum showing both the channel power magnitude $\left(M_{C P}\right)$ at a characteristic frequency $\left(f_{C}\right)$ and the $M_{C P}$ at switching frequency $\left(f_{S W}\right)$ can be obtained.

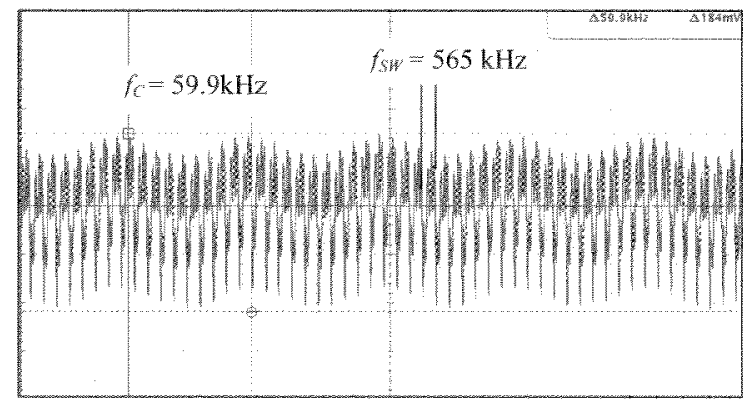

(a)

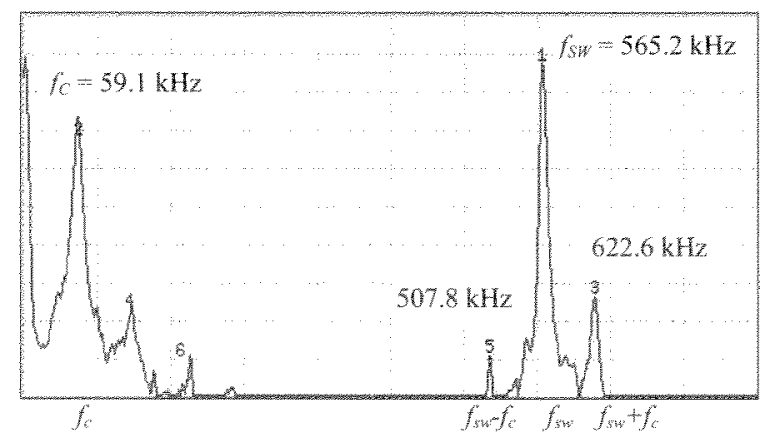

(b)

Migure 1: A typical case of AM-type oscillation in its early stage, indicating in a timedomain waveform (a) and a power spectrum (b).

\footnotetext{
* This study was part of DC/DC converter stabihty study funded by NASA Electronic Parts and Packaging Program (NEPP). The author was an employee of Dell Services Federal Govemment (DSFG) on site of GSFC.
} 
Figure 1 (a) and (b) show a typical case of AM-type oscillation in its early stage, which indicate $f_{C}$ and $f_{S W}$ on both input noise Time-domain waveform and power spectrum. Figure 2 shows a typical FM-type severe oscillation. Figure 2(a) indicates the $f_{S W}$ and frequency swing $\left(\Delta f_{S W}\right)$ in time-domain waveform, while Figure 2(b) shows the spectrum with $f_{C}, f_{S W}$, and sideband frequencies $\left(f_{S W} \pm x f_{C}\right)$. Note that each of the sideband frequencies changes at increment of $f_{C}$, which clearly implies that the characteristic frequency $\left(f_{C}\right)$ is a carrier frequency, and in this case, is a representative of the oscillation frequency $(f o s)$.

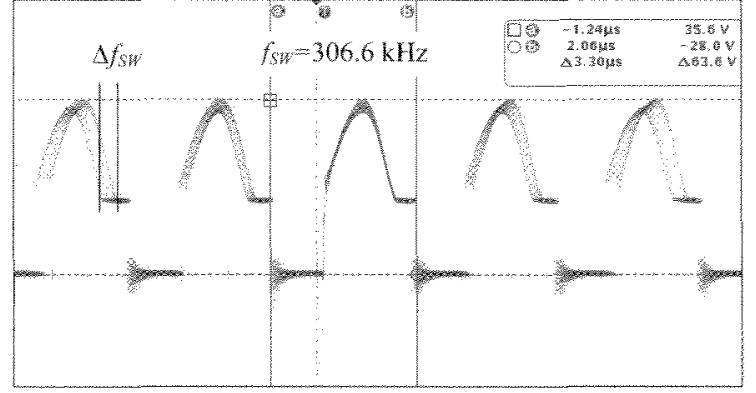

(c)

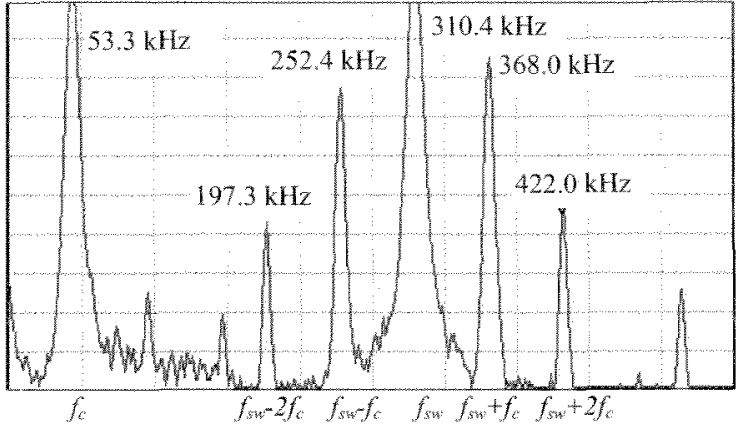

(d)

Figure 2: A typical severe FM-type oscillation showing as time-domain waveform (a) and power spectrum (b).

Oscillation mechanism of the DC/DC converter was analyzed, based on feedback loop control theory [3]. A series of oscillations were simulated using an oscillation simulator with a QV24-525 converter, by altering parameters of $\mathrm{RC}$ components within the feedback control loop. The study proved that the oscillation occurred at phase-crossover frequency $\left(f_{p c o}\right)$ in a Bode Plot where phase shift came to $0^{\circ}\left(360^{\circ}\right)$ as shown in Figure 3(a). In order to prove the existence of the oscillation frequency, a corresponding power spectrum in Figure 3(b) was taken at the same condition as the Bode plot was obtained. The crossover frequency of $98.9 \mathrm{kHz}$ measured in the Bode plot was detected in the power spectrum as $94.6 \mathrm{kHz}$. To further investigate the correlation between the frequencies of the two measurement methods, 18 phase-crossover frequencies $\left(f_{p c o}\right)$ and 18 corresponding channel power frequencies $\left(f_{c p}\right)$ were measured simultaneously while adjusting the simulator to generate 18 incremental oscillation noise levels. The test results in

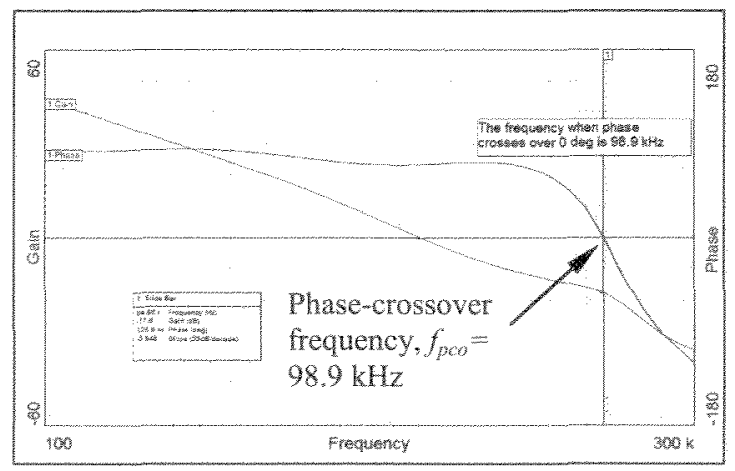

(a)

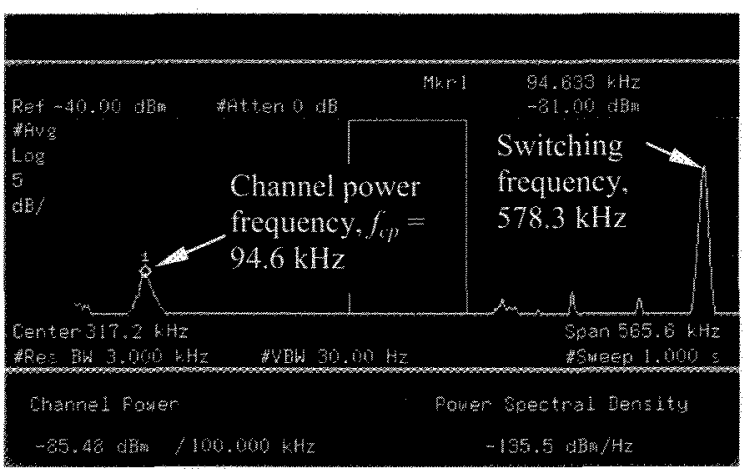

(b)

Figure 3: The Phase-Crossover Freqency $\left(f_{p c o}\right)$ indicated in a Bode plot (a) can be measured in a power spectrtum (b) as a Channal Power Frequency $\left(f_{c p}\right)$. 
Figure 4(a) showed that the two frequencies had strong relationship with a correlation coefficient of $r=0.9453$ and with the fact that $89.4 \%$ of power spectrum readings were accounted for by the Bode plot readings. Figure $4(\mathrm{~b})$ represented correlation of the frequencies for a MFL2812S DC/DC convertor, which showed even higher correlation for the two measurement methods. In general, the feedback loop oscillation frequency detected by using a spectral analysis method was determined to be in a high frequency range, unique for every individual type of the converters, and in strong linear correlation with the phase-crossover frequency presented in a Bode plot of the gain/phase margin measurement method [1][4].

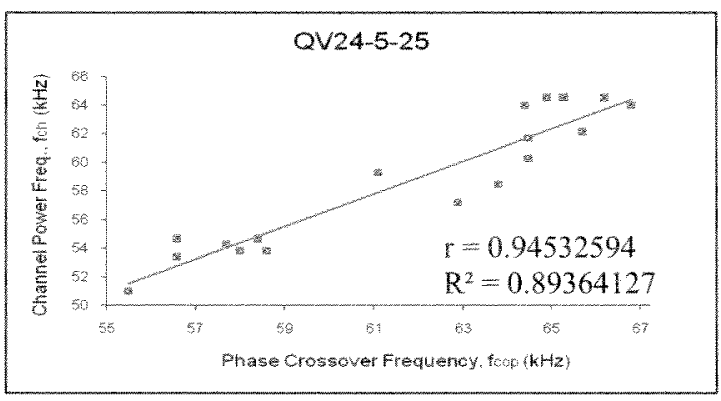

(a)

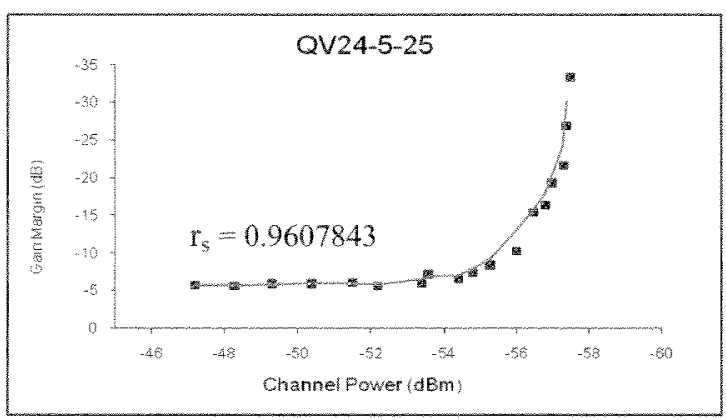

(c)

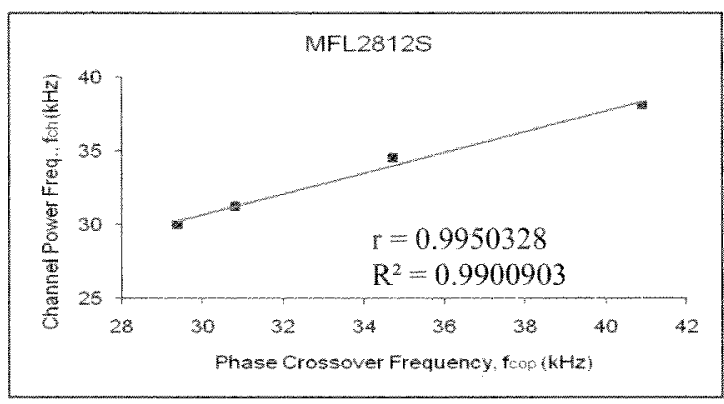

(b)

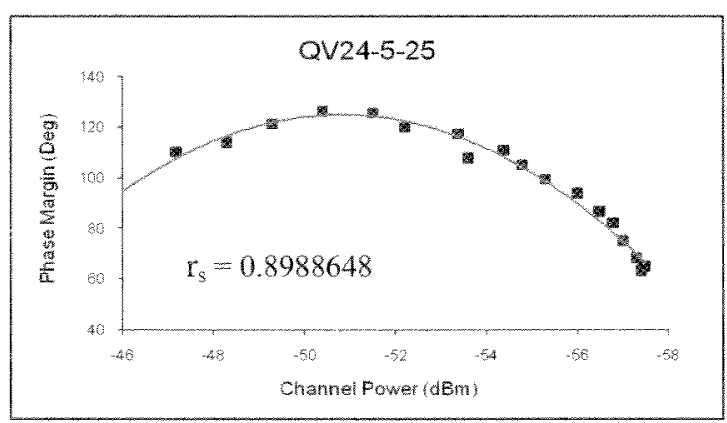

(d)

Figure 4: Correlations between phase-crossover frequency $\left(f_{p c o}\right)$ in a Bode Plot and channel power frequencies $\left(f_{c p}\right)$ for QV24-5-25 (a) and MFL2812S (b), and correlations between the channel power of power spectrum and gain margin (c) and phase margin (d) / for QV24-5-25.

For DC/DC converter instability diagnosis, the channel power magnitude $\left(M_{C P}\right)$ at a specified characteristic frequency $\left(f_{C}\right)$ from input noise power spectrum of a DC/DC converter could be used as a precise stability level indicator. It was found that the $M_{C P}$ at characteristic frequency $\left(f_{c}\right)$ could truly respond to a very small change of oscillation noise merged in the input voltage noise. Tests were conducted to determine if the $M_{C P}$ in the input noise power spectrum had strong correlation with the gain/phase margins measured under the same test conditions. Figures $4(c)$ and 4(d) illustrated the relationship between the channel power and gain and phase margins, which were obtained by using the oscillation simulator. Because the correlation appeared to be non-linear, Rank Order Correlation Coefficient $\left(r_{s}\right)$ was utilized to describe how the channel power (in $\mathrm{dBm}$ ) increased (or decreased) with changes of gain and phase margins. The test curves indicated the clear trends that the $M_{C P}$ decreased while the gain and phase margins increased (or decreased) accordingly with quite large $r_{s}$ values equal to 0.9608 for gain margin 
and 0.8989 for phase margin. In fact, the lower $r_{s}$ value simply meant that there was a larger portion of negative relationship between the two parameters, compared to the positive relationship shown in Figure 4(c). The $M_{C P}$ contains total average noise energy of the oscillation within a specified frequency range, so that it makes the channel power reading more stable and more sensitive to oscillation in early stage than the pulse amplitude in a Fourier spectrum as observed in this experiment.

In conclusion, the oscillation frequency can be recognized in Bode plot as zero-phase frequency $\left(f_{z p}\right)$ which is the fundamental of the early oscillation detection technique. The Characteristic frequency $\left(f_{c}\right)$ is defined for the spectrum analysis method, thus the $f_{c}$ represents the $f_{z p}$. Any amount of increase in $M_{C P}$ from their nominal levels at $f_{c}$ and at $f_{s w}$ sidebands could indicate an ongoing oscillation and can be utilized to determine the types, intensity, and change tendency of the oscillation. This technique shows significant advantages over gain/phase analysis method in terms of detection sensitivity, real-time monitoring capability, and test probing flexibility. In addition, it potentially provides a powerful tool to flight DC/DC converter health monitoring and fault diagnosis.

\section{REFERENCES}

1. Bright L. Wang, DC/DC Converter Stability Testing Study, NASA/CR-2008-214163, 2008, National Information Service, 5282 Port Royal Road, Springfield, VA 22161; NASA Center for Aerospace Information, 7115 Standard Dr., Hanover, MD 21076; http://nepp.gsfc.nasa. gov.

2. Lloyd Temes, Theory and Problems of Electronic Communication, Schaum's Outline Series, McGraw-Hill, New York (ISBN: 0-07-063495-5), 1979.

3. Abraham I. Pressman, Switching Power Supply Design, McGraw-Hill, New York (ISBN: 007-050806-2), 1991, pp. 427-470.

4. Bright L. Wang, Early Oscillation Detection for Hybrid DC/DC Converters, NASA Electronic Parts Program (NEPP) Report, QSS-05-S-246, January 2009; http://nepp.gsfe. nasa. gov.

5. George C. Chrysis, High-Frequency Switching Power Supplies, Theory and Design, McGraw-Hill, New York, 1984, pp. 233-244.

6. Marty Brown, Power Supply Design Cookbook, Motorola, 2001, pp. 145-167.

7. R. D. Middlebrook, Input Filter Considerations in Design and Application of Switching Regulators, IEEE Industry Applications Society Annual Meeting (IEEE Publication 76CH1122-1-IA), 1976 Record, pp. 336-382.

8. Fred C. Lee and Yuan Yu, Input-Filter Design for Switching Regulators, IEEE Transactions on Aerospace and Electronic Systems, Vol. AES-15, No.5, 1979, pp.627-634.

9. Venable Industries, New Techniques for Measuring Feedback Loop Transfer Functions in Current Mode Converters, Venable Technical Paper \#8.

10. SynQor, "Input System Instability, Application Note" PQ-00-05-1, Rev 01, 2000.

11. D. H. Venable, "Testing Power Sources for Stability", Production Applications, Technical Paper\#1, www.vanableindustries.com. 2000.

12. D. H. Venable, "Source Load Interactions in Multi-Unit Power Systems", AIAA-94-4235CP, Venable Industries, Inc, 1994

13. R. Ridley, "Loop Gain Measurement", Switching Power Magazine, 2005, pp. I-3 